\title{
Land of hope and dreams
}

\section{Selection of life science and translational medicine literature \\ by Marco Confalonieri}

Why two individuals with the same mutation may
have two different phenotypes?
The principles of genetics have been repeatedly subverted in the last years, especially from the conclusion of the so-called human genome project. The continuous changing of the basis of the universe of genomic knowledge hinders the routine use of commercial genetic tests that promised to reveal users' risk for various illness. As a practical physician, I am hopefully waiting for bringing DNA study at patient bedside, but recently some scientists stated personal genome sequencing limits, so questioning its value (1). In fact, one of the latest acquisition of genomics is the deep difference between the presence of a genetic risk factor for a specific disorder and the clinical manifestation of it. In other words, there is no defined health destiny for any person, because there is always a strong random component in anything that happens to each individual. Nevertheless, even if DNA study does not perfectly determine which disease an individual may develop in the future, may it become a useful tool to assess a disease risk from a practical point of view? To get an insight in this key point it should have been necessary to study tons of identical twins or thousands of people with the same pathologic genetic mutations. Luckily, in the recent decades most findings on genome function were firstly studied through the DNA of the worm Caenorhabditis Elegans (Figure 1), a highly cited animal model because at least 3 recent Nobel Prize on Physiology or Medicine were won thanks to it. So, by studying $C$. Elegans, the group of Andrew Fraser at the University of Toronto (2) found a possible reason why the same mutation does not results in the same phenotype or disease. They were determined for a 7 year-period to follow a genome screening of 1,400 gene mutations in two strains of $C$. Elegans seeing what happened when they knocked down each gene. The researchers found that about $20 \%$ of gene mutations had variations in the phenotype expression between worms. Particularly, they found that when a mutation led to a milder phenotype in one animal, its associated gene was expressed at a lower level. The measure of gene expression, after gene sequence, is likely to be applicable in the next future to human studies that analyze phenotype variations to predict which subjects among those having the same pathologic genetic variation may develop a determined gene-related disease in a milder or more severe form. Obviously, gene expression is not the only influencing phenotype factor, but further studies could point the way toward specific risk factors and gene interactions that affect phenotypes, which could then be specifically tested in humans. Next genera-

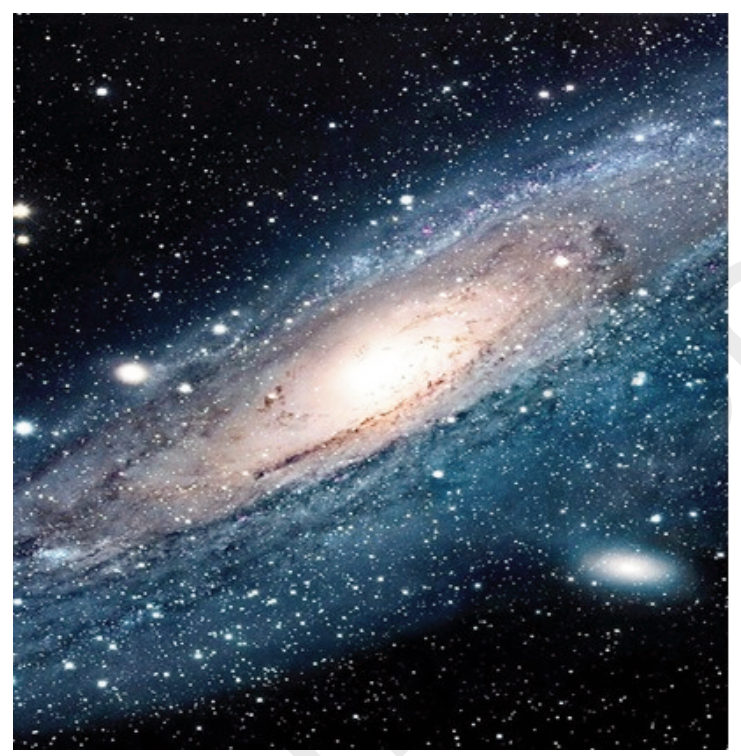

tion medicine will probably need the measurement of gene expression combined with gene sequencing to exactly predict how the same predisposition for a disorder may influence differently the expression of illness severity in different subjects with the same genetic variation. So, the study by Fraser et al. might represent a very important step to save the power of personal genomic study in clinical medicine.

\section{1) The predictive capacity of personal genome se-} quencing

Roberts JR, Vogelstein JT, Parmigiani G, Kinzler KW, Vogelstein B, Velculescu VE

Sci Transl Med 2012;4:133ra58

New DNA sequencing methods will soon make it possible to identify all germline variants in any individual at a reasonable cost. However, the ability of wholegenome sequencing to predict predisposition to common diseases in the general population is unknown. To estimate this predictive capacity, we use the concept of a "genometype". A specific genometype represents the genomes in the population conferring a specific level of genetic risk for a specified disease. Using this concept, we estimated the maximum capacity of whole-genome sequencing to identify individuals at clinically significant risk for 24 different diseases. Our estimates were derived from the analysis of large numbers of monozygotic twin pairs; twins of a pair share the same genometype and therefore identical genetic risk factors. Our analyses indicate that: (i) for 23 of the 24 diseases, most of the individuals will receive negative test results; (ii) these negative test results will, in general, not be very informative, because the risk of developing 19 of the 24 diseases in those who test negative will still be, at minimum, 50 to $80 \%$ of that in the general population; and (iii) on the positive side, in the best-case scenario, more than $90 \%$ of 


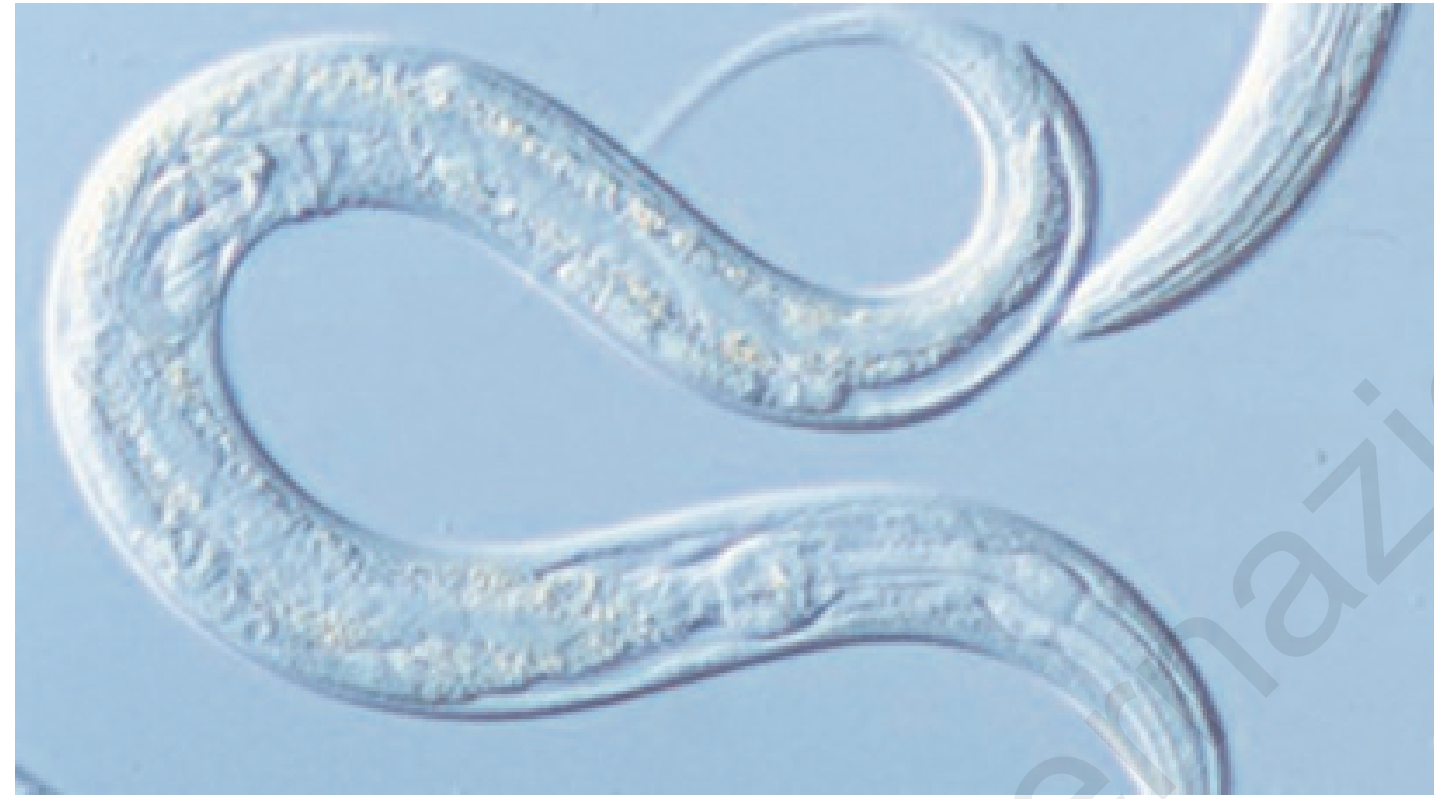

Figure 1 - Caenorhabditis Elegans.

tested individuals might be alerted to a clinically significant predisposition to at least one disease. These results have important implications for the valuation of genetic testing by industry, health insurance companies, public policy-makers, and consumers.

\section{2) Natural variation in gene expression modulates} the severity of mutant phenotypes

Vu V, Verster AJ, Schertzberg M, Chuluunbaater T, Spensley M, Pajkic D, Hart GT, Moffat J, Fraser AG Cell 2015;162:391-402

Many mutations cause genetic disorders. However, two people inheriting the same mutation often have different severity of symptoms, and this is partly ge- netic. The effects of genetic background on mutant phenotypes are poorly understood, but predicting them is critical for personalized medicine. To study this phenomenon comprehensively and systematically, we used RNAi to compare loss-of-function phenotypes for $\sim 1,400$ genes in two isolates of $C$. elegans and find that $\sim 20 \%$ of genes differs in the severity of phenotypes in these two genetic backgrounds. Crucially, this effect of genetic background on the severity of both RNAi and mutant phenotypes can be predicted from variation in the expression levels of the affected gene. This is also true in mammalian cells, suggesting it is a general property of genetic networks. We suggest that differences in the manifestation of mutant phenotypes between individuals are largely the result of natural variation in gene expression. 